\title{
Impacto da análise do 'wavefront' na refratometria de pacientes com ceratocone
}

\author{
Impact of the wavefront analysis \\ in refraction of keratoconus patients
}

Renato Ambrósio Junior ${ }^{1,2,3}$, Diogo Leitão Caldas ${ }^{2,3,4}$, Renata Siqueira da Silva ${ }^{2,3}$, Leonardo Nogueira Pimentel ${ }^{2,3}$, Bruno de Freitas Valbon ${ }^{2,3,4}$

\section{ResUmo}

Objetivo: Verificar se a aberrometria ocular total (análise da frente de onda ou 'wavefront') possibilita melhora na acuidade visual corrigida (AVc) com lentes esferocilíndricas, obtida com a refratometria manifesta em casos de ceratocone com algum grau de intolerância ao uso de lentes de contato. Métodos: Os prontuários de 46 pacientes ( 89 olhos), referidos com diagnóstico de ceratocone e intolerantes ao uso de lentes de contato, submetidos ao exame de aberrometria ocular total seguido de refração manifesta, foram estudados de forma retrospectiva. A AVc (logMAR) com a correção existente antes do exame foi comparada com a obtida com a nova refração manifesta, realizada considerando-se os dados objetivos da aberrometria. $O$ teste não-paramétrico de Wilcoxon para amostras pareadas foi utilizado para verificação de diferenças estatisticamente significantes na AVc. Resultados: Houve uma melhora estatisticamente significante na AVc com a nova refração manifesta $(\mathrm{p}<0,0001)$. A AVc média passou de 0,37 ou 20/47 (variando entre 1,3 e 0 ; desvio padrão[DP] $=0,25$ ) com a refração prévia para 0,23 ou $20 / 34$ (variando entre 1 e 0,$1 ; \mathrm{DP}=0,21$ ). Cinquenta e dois olhos $(58,4 \%$ ) de 28 pacientes apresentaram melhora na AVc com a nova refração. A melhora média foi de 0,13 logMAR (1,3 linhas na tabela de Snellen), variando entre nula e 0,6 (6 linhas), com desvio padrão de 0,16 . Oito pacientes apresentaram anisometropia significativa que limitou a prescrição de óculos em um dos olhos. Conclusão: A aberrometria facilitou a refratometria, determinando melhora significativa na acuidade visual corrigida com as lentes esfero-cilíndricas de pacientes com ceratocone intolerantes ao uso de lentes de contato. A anisometropia foi um fator limitante na prescrição de óculos.

Descritores: Aberrações de frente de onda da córnea; Ceratocone; Lentes de contato; Refração ocular; Acuidade visual

\footnotetext{
'Pontifícia Universidade Católica do Rio de Janeiro - PUC-Rio - Rio de Janeiro (RJ), Brasil;

${ }^{2}$ Instituto de Olhos Renato Ambrósio - Rio de Janeiro (RJ), Brasil;

${ }^{3}$ Rio de Janeiro Corneal Tomography and Biomechanics Study Group - Rio de Janeiro (RJ), Brasil;

${ }^{4}$ Universidade Federal Fluminense - UFF - Rio de Janeiro (RJ), Brasil.
}

Trabalho realizado no Instituto de Olhos Renato Ambrósio - Rio de Janeiro (RJ), Brasil. 


\section{INTRODUÇÃO}

C eratocone é uma distrofia ectásica não inflamatória de caráter progressivo, caracterizada por afinamento e encurvamento da córnea, com protrusão cônica central ou paracentral ${ }^{(1,2)}$. Tais mudanças na geometria da córnea induzem astigmatismo irregular e miopização, determinando prejuízo na acuidade visual $^{(3,5)}$. A doença ocorre em todas as raças, é tipicamente bilateral, mas assimétrica, apresenta uma incidência de 1:2.000 na população geral e tem discreta preponderância no sexo feminino ${ }^{(1)}$. Diversos estudos apontam que o ceratocone afeta significativamente a qualidade de vida do paciente ${ }^{(6,1)}$, além de ser uma das causas mais freqüentes de transplante de córnea ${ }^{(12-17)}$.

A reabilitação visual de pacientes com ceratocone é dada inicialmente com óculos. Entretanto, trata-se de um desafio para a refratometria, devido à presença de astigmatismos elevados e não regulares. A presença de elevados índices de irregularidades ópticas (HOA - high order aberrations) pode determinar que a correção esfero-cilíndrica de óculos tradicionais seja insatisfatória. Neste cenário, lentes de contato (LC) especiais são indicadas ${ }^{(18-21)}$. Tradicionalmente, a ceratoplastia ou transplante de córnea eram as opções de cirurgias terapêuticas indicadas em situações de intolerância na adaptação de LC. Entretanto, novas técnicas cirúrgicas como a promoção de ligações covalentes e reticulação do colágeno corneano com radiação ultravioleta e riboflavina (crosslinking) ${ }^{(22-25)}$, o implante de segmentos de anéis intraestromais ${ }^{(26-31)}$, lentes fácicas ${ }^{(32-33)}$, e técnicas de fotoablação ${ }^{(34-38)}$ são capazes de adiar ou mesmo evitar o transplante. Tais procedimentos podem ser indicados mais precocemente e de forma eletiva, sendo fundamental uma propedêutica avançada para confirmar a indicação, bem como para o planejamento cirúrgico.

Nas últimas décadas, observou-se uma verdadeira revolução nos métodos diagnósticos em córnea, destacando-se o advento da topografia de reflexão dos discos de Placido $^{(39,42)}$, tomografia com reconstrução 3-D ${ }^{(43-46)}$, estudo biomecânico da córnea in vivo ${ }^{(47-52)}$ e a aberrometria ocular ${ }^{(53,59)}$. A análise da frente de onda (wavefront) ou aberrometria ocular total permite a caracterização das aberrações ópticas do olho de forma mais detalhada e complexa que a tradicionalmente realizada. As aberrações ópticas são decompostas matematicamente, sendo os polinômios de Zernike a forma mais popular ${ }^{(60-62)}$, pois as aberrações de ordem baixa correspondem aos termos da refratometria esfero-cilíndrica tradicional (astigmatismo e desfoco [miopia e hipermetropia]).
A aberrometria ocular tem seu papel destacado em casos de ceratocone para diagnóstico ${ }^{(57,63-66)}$, estadiamento $^{(67)}$, e avaliação do tratamento realizado ${ }^{(65,68-}$ ${ }^{71)}$. O presente estudo pretende avaliar se os dados objetivos com a aberrometria ocular total podem auxiliar na refratometria manifesta, de modo a proporcionar melhor acuidade visual corrigida com óculos (AVc).

\section{MÉTODOS}

Os prontuários de pacientes com diagnóstico de ceratocone, com histórico de algum grau de intolerância ao uso de LC, submetidos ao exame de aberrometria seguido de refratometria foram selecionados para estudo retrospectivo. O estudo seguiu os critérios e recomendações da Declaração de Helsinki e o estudo fez parte de um protocolo aprovado por comitê Universitário de ética para pesquisas em humanos. Cirurgias oculares prévias ou quaisquer doenças oculares diferentes do ceratocone foram critérios de exclusão.

$\mathrm{O}$ exame de aberrometria foi realizado em condições dinâmicas de acomodação e reflexo pupilar sob estratégia de fogging (ou borramento da mira), utilizando-se o sistema "iTrace" (Tracey Technologies, Houston, EUA) que realiza aberrometria por meio de raytracing de pontos individuais de laser projetados na retina, de forma integrada à topografia de Plácido. Duas sequências de 128 , num total de 256 pontos projetados dentro da abertura pupilar (Figura 1) foram utilizadas para cada exame.

A posição de cada ponto na retina é identificada de modo que a reconstrução do Retinal Spot Diagram (Figura 2A) possibilite a visualização da função de espalhamento de um ponto (FEP ou Point Spread Function - Figura 2B). Um olho sem aberrações ópticas teria uma convergência de todos os pontos para o centro do RSD. O desvio de cada ponto é calculado para reconstrução da frente de onda de acordo com os polinômios, e Zernike sob a forma de elevação (micra) de RMS (Root Mean Square) (Figura 3). A partir dos termos de baixa ordem (segunda ordem, Z2), é possível se determinar os componentes esfero-cilíndricos em Dioptria. A zona óptica de $4 \mathrm{~mm}$ é classicamente utilizada para cálculo da autorrefração dos aberrômetros ${ }^{(4,72)}$. O RMS total, de baixa ordem, de alta ordem, do desfoco, do astigmatismo (e seu eixo), das aberrações de alta ordem, da aberração esférica, do coma (e seu eixo) e do trefoil (e seu eixo) foram calculados para $4 \mathrm{~mm}$ e tabulados, juntamente com a autorrefração.

A AVc obtida com a correção utilizada na oca- 
sião do exame foi convertida em logMAR de modo a possibilitar análise comparativa ${ }^{(73,74)}$. Após o exame, nova refração foi obtida com base nos dados objetivos encontrados e a nova $\mathrm{AV} c$ foi tabulada. $\mathrm{O}$ teste não-paramétrico de Wilcoxon para amostras pareadas foi utilizado para verificação de diferenças estatisticamente significantes na $\mathrm{AVc}$ e entre a autorrefração da aberrometria e a refratometria manifesta.

O teste não-paramétrico de Spearman foi utilizado para verificar se existiram correlações significativas entre a melhora da AVc e os índices de RMS encontrados, bem como entre o equivalente esférico, desfoco e cilindro da autorrefração da aberrometria e a refratometria manifesta.

\section{Resultados}

Os prontuários de 46 pacientes (89 olhos) foram avaliados. Todos os pacientes foram encaminhados para exame de aberrometria por apresentarem diagnóstico de ceratocone que foi confirmado pela topografia de Plácido. Todos os pacientes apresentavam-se com refração prévia realizada por oftalmologista e óculos, que foram prescritos entre 1 e 10 meses antes do exame (média de 3,4 meses; $\mathrm{DP}=2,14$ ). Intolerância absoluta a LC foi observada em 16 pacientes (35\%), sendo que todos os pacientes apresentavam queixas relacionadas com LC e dificuldades na adaptação.

Observou-se melhora estatisticamente significantiva na $\mathrm{AVc}$ com a nova refração manifesta $(\mathrm{p}<0,0001)$. A AVc média passou de $0,37 \log$ MAR ou 20/47 (variando entre 1,3 e 0; desvio padrão[DP]=0,25) com a refração prévia para $0,23 \log$ MAR ou 20/34 (variando entre 1 e 0,$1 ; \mathrm{DP}=0,21$ ). A melhora média foi de $0,13 \log$ MAR (1,3 linhas na tabela de Snellen), variando entre nula e 0,6 (6 linhas), com desvio padrão de 0,16.

Não houve diferença significante entre o grau esférico $(\mathrm{p}=0,83)$, cilindro $(\mathrm{p}=0,79)$, equivalente esférico $(=0,52)$ e eixo do cilindro (radiano, $p=4,39)$ da refração manifesta e da autorrefração obtida por meio da aberrometria. Houve uma forte correlação estatisticamente significante para todas as variáveis $(\mathrm{p}<0,01)$. Os coeficientes de correlação de Spearman (rho) foram de 0,72 para o grau esférico, 0,61 para o grau do cilindro, 0,79 para o equivalente esférico e 0,43 para o eixo do cilindro (radiano).

Observou-se correlações significativas entre a melhora da AVc e o índice RMS total $(\mathrm{p}=0,038$; rho=0,29), RMS de baixa ordem $(\mathrm{p}=0,039$; $\mathrm{rho}=0,29)$, RMS do desfoco $(\mathrm{p}=0,03$, rho $=0,30)$ e o RMS da aberra-

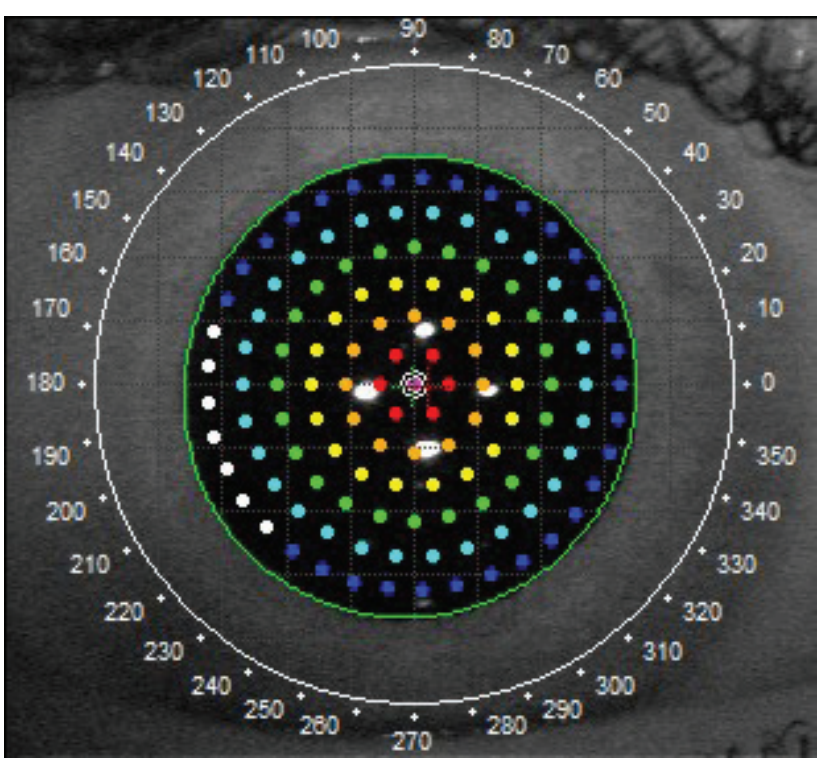

Figura 1: Escaneamento dentro da pupila

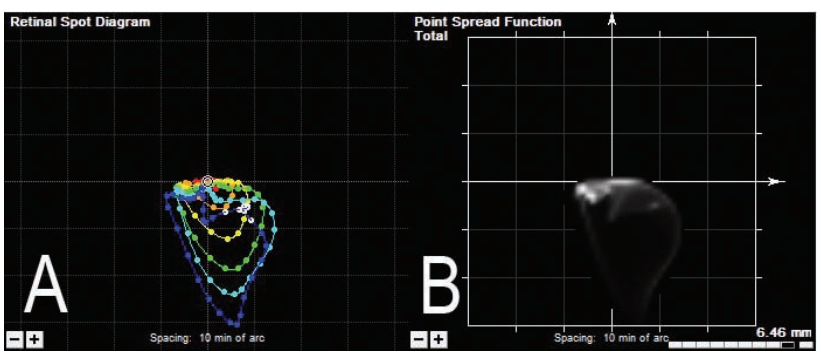

Figura 2: Diagrama dos pontos na retina (A) e função de espalhamento do ponto (FEP - B)

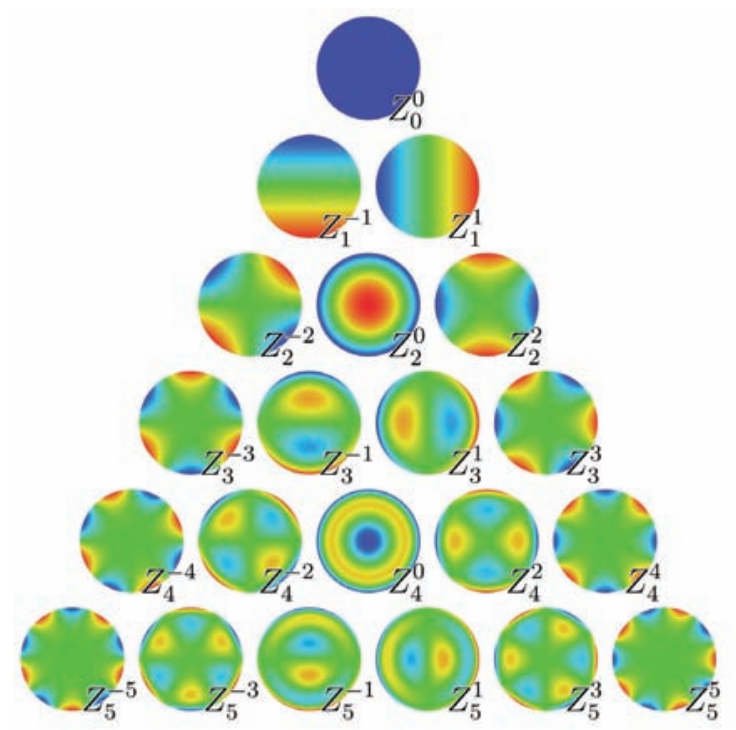

Figura 3 - Polinomios de Zernike; a terceira linha (Z2) corresponde aos termos esfero-cilíndricos: desfoco - $Z_{2}^{0}$ e astigmatismo - $Z^{-2}{ }_{2} \mathrm{e}$ $\mathrm{Z}^{2}{ }_{2}$ 


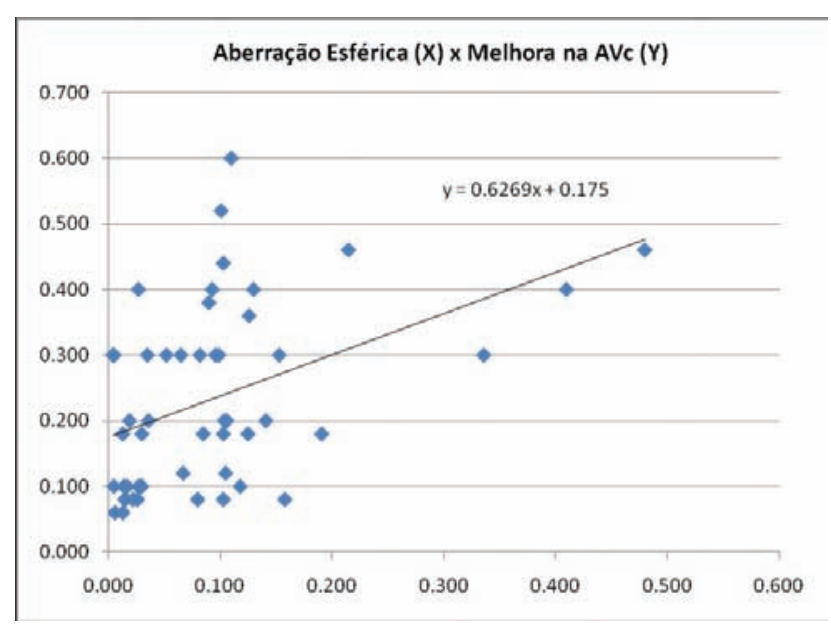

Figura 4: Correlação entre a melhora da AVc e o valor absoluto da aberração esférica em $4 \mathrm{~mm}$ de zona óptica; rho=0,43

ção esférica ( $\mathrm{p}=0,0018$, rho=0,43 - Figura 4).

Cinquenta e dois olhos $(58,4 \%)$ de 28 pacientes apresentaram melhora na AVc com a nova refração. Oito pacientes (29\% dos que apresentaram melhora da AVc) apresentaram anisometropia significativa que limitou a prescrição de óculos em um dos olhos. Óculos foram prescritos para 20 pacientes $(43,5 \%)$, com reabilitação visual satisfatória, possibilitando adiar a indicação de cirurgia sem necessidade de insistir com o uso de LC.

\section{DıscussÃo}

A aberrometria ou análise da frente de ondas (wavefront) é um novo recurso propedêutico, introduzido classicamente para programação de cirurgia refrativa personalizada com excimer laser ${ }^{(75-78)}$. Entretanto, aplicações para diagnóstico e caracterização da óptica ocular, bem como para auxiliar na refratometria foram descritas $^{(4,59,79,80)}$. Diversos mapas e índices são possíveis serem calculados com base nos dados do exame (Figura 5). É possível calcular tais índices para zonas ópticas menores que a área total da pupila examinada. A partir da FEP, é possível reconstruir uma simulação da imagem retiniana, utilizando-se cálculos de convolução de quaisquer objetos. Tipicamente utiliza-se a letra $\mathrm{E}$ que pode ter sua frequência espacial ajustada. Tanto a FEP, como as imagens de convolução podem ser calculadas para todas as aberrações ou para cada aberração individualmente ou agrupadas. A convulução considerandose as aberrações de alta ordem (HOA total - high order aberrations) permite a simulação da visão obtida com lentes esfero-cilíndricas (Figura 5).

Diversos métodos podem ser utilizados para a

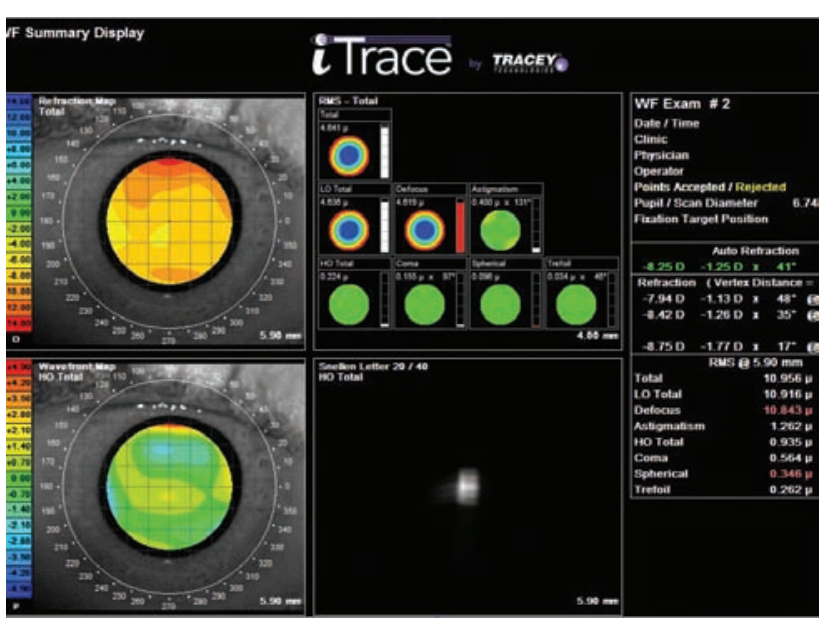

Figura 5: Sumário WF de uma paciente que passou a AVc de 20/ 60 para 20/25 com nova Refração: $-8,00-1,25$ x $43^{\circ}$

aberrometria $^{(4)}$, destacando-se o Hartmann-Shack, Tserning, esquiescopia rotacional e raytracing de pontos individuais. $\mathrm{O}$ escaneamento de pontos individuais possibilita a capacidade de medidas em olhos com distorções ópticas mais acentuadas, sendo uma vantagem em caso de ceratocone ${ }^{(81)}$.

A aberrometria pode fornecer dados para confecção de lentes de contato gelatinosas personalizadas com correção para as aberrações de alta ordem ${ }^{(82,83)}$. Tais alternativas poderão ser utilizadas comercialmente em breve, mas ainda não estão disponíveis no mercado brasileiro no tempo da escrita deste artigo. A alternativa de óculos com correção das aberrações de alta ordem também é interessante e já é uma realidade (http:// www.izonlens.com), mas as limitações relacionadas com a distância vértice são importantes.

Neste estudo, verificamos que a aberrometria foi relevante no sentido de facilitar a refratometria de casos de ceratocone, tendo significativo impacto para melhorar a AVc de pacientes com algum grau de intolerância ao uso de LC que seriam candidatos a cirurgia. Alguns pacientes tinham última refração entre 6 e 10 meses e a progressão da doença pode ser responsável pela baixa na AVc. Entretanto, todos os pacientes vinham encaminhados por outro oftalmologista que teria descartado a possibilidade de nova refratometria em consulta recente. As informações objetivas obtidas com a aberrometria foram utilizadas como base para a refração subjetiva, que é uma etapa fundamental independente da qualidade dos exames objetivos realizados. Adicionalmente, não houve diferenças significantes entre a refração manifesta e a autorrefração obtida por meio da aberrometria, bem como houve forte correlação entre tais parâmetros. A anisometropia foi, entretan- 
to, um fator limitante para a prescrição de óculos, ocorrendo de forma significante em $29 \%$ dos que apresentaram melhora da AVc.

Existe um futuro promissor para a aberrometria em relação a seu reconhecimento como método propedêutico na Oftalmologia. Certamente o papel da análise da frente de onda (wavefront) vai além da cirurgia refrativa personalizada, que permanecerá como uma das aplicações mais importantes. Por exemplo, dados da aberrometria podem auxiliar na escolha da asfericidade da lente intraocular (LIO) na cirurgia da catarata ${ }^{(84-86)}$. No presente estudo, demonstramos a relevância da aberrometria para refratometria de pacientes com ceratocone. A melhora da AVc obtida em 58,4\% dos casos justifica que a refratometria deve ser efetivamente tentada antes de se considerar a indicação absoluta de cirurgia, ou mesmo para diminuir a dependência ao uso de LC. A Aberrometria deve ser considerada para fornecer dados objetivos para melhorar ou facilitar a refração manifesta de pacientes com ceratocone.

\section{Abstract}

Objective: To verify if the total ocular aberrometry (wavefront analysis) facilitates manifest refraction and improvement in best spectacle distance corrected visual acuity (BSCDVA) with sphero-cylindrical lenses, in keratoconus cases with some degree of contact lenses intolerance.Methods: Retrospective chart review of 46 patients (89 eyes) referred with keratoconus and contact lenses intolerance was performed. Ocular aberrometry with ray tracing was followed by manifest refraction. BSCDVA (logMAR) with the previous correction was compared with the one obtained based on the wavefront autorefraction. The nonparametric test of Wilcoxon for paired samples was used to test statistically significant differences in BSCDVA. Results: There was a statistically significant improvement in BSCDVA with the new manifest refraction $(p<0,0001)$. The average BSCDVA changed from 0,37 or 20/47 (varying between 1,3 and 0; standard deviation $[S D]=0,25)$ with previous refraction to 0,23 or 20/34 (varying between 1 and 0,$1 ; S D=0,21$ ). 52 eyes $(58,4 \%)$ of 28 patients showed improvement in $B S C D V A$ with the new refraction. The average improvement was 0,13 $\log M A R$ (1,3 lines on Snellen chart), varying between zero and 0,6 (6 lines), with standard deviation of 0,16. Eight patients had significant anisometropia that limited the prescription of glasses. Conclusion: Aberrometry facilitated the refraction, determining significant improvement in visual acuity with sphero-cylindrical lenses in keratoconus patients intolerant to contact lenses. The anisometropia was a limiting factor in the prescription of glasses.

Keywords: Corneal wavefront aberration; Keratoconus; Contact lenses; Refraction, ocular; Visual acuity

\section{REFERÊNCIAS}

1. Rabinowitz YS. Keratoconus. Surv Ophthalmol. 1998;42(4):297-319. Review.

2. Krachmer JH, Feder RS, Belin MW. Keratoconus and related noninflammatory corneal thinning disorders. Surv Ophthalmol. 1984;28(4):293-322. Review.

3. McGhee CN. 2008 Sir Norman McAlister Gregg Lecture: 150 years of practical observations on the conical cornea-what have we learned? Clin Experiment Ophthalmol. 2009;37(2):160-76.

4. Maeda N. Clinical applications of wavefront aberrometry - a review. Clin Experiment Ophthalmol. 2009;37(1):118-29. Review.

5. Radhakrishnan H, O'Donnell C. Aberrometry in clinical practice: case series. Cont Lens Anterior Eye. 2008;31(4):207-11.

6. Szczotka LB, Barr JT, Zadnik K. A summary of the findings from the Collaborative Longitudinal Evaluation of Keratoconus (CLEK) Study. CLEK Study Group. Optometry. 2001;72(9):574-84

7. Moreira LB, Alchieri JC, Belfort Júnior R, Moreira H. Aspectos psicossociais do paciente com ceratocone. Arq Bras Oftalmol. 2007;70(2):317-22.

8. Kymes SM, Walline JJ, Zadnik K, Sterling J, Gordon MO; Collaborative Longitudinal Evaluation of Keratoconus Study Group. Changes in the quality-of-life of people with keratoconus. Am J Ophthalmol. 2008;145(4):611-7.

9. Kymes SM, Walline JJ, Zadnik K, Gordon MO; Collaborative Longitudinal Evaluation of Keratoconus study group. Quality of life in keratoconus. Am J Ophthalmol. 2004;138(4):52735. Comment in: Am J Ophthalmol. 2004;138(4):637-8.

10. Giedd KK, Mannis MJ, Mitchell GL, Zadnik K. Personality in keratoconus in a sample of patients derived from the internet. Cornea. 2005;24(3):301-7.

11. de Freitas Santos Paranhos J, Avila MP, Paranhos A Jr, Schor P. Evaluation of the impact of intracorneal ring segments implantation on the quality of life of patients with keratoconus using the NEI-RQL (National Eye Institute Refractive Error Quality of life) instrument. Br J Ophthalmol. 2010;94(1):101-5.

12. Castroviejo R. Keratoplasty in treatment of keratoconus. Arch Ophthal. 1949;42(6):776-800, illust.

13. Lindquist TD, McGlothan JS, Rotkis WM, Chandler JW. Indications for penetrating keratoplasty: 1980-1988. Cornea. 1991;10(3):210-6.

14. Haamann P, Jensen OM, Schmidt P. Changing indications for penetrating keratoplasty. Acta Ophthalmol (Copenh). 1994;72(4):443-6.

15. Flowers CW, Chanq KY, McLeod SD, Irvine JA, McDonnell PJ, Rao N, Smith RE. Changing indications for penetrating keratoplasty, 1989-1993. Cornea. 1995;14(6):583-8. Comment in: Cornea. 1997;16(1):120-1. 
16. Cursiefen C, Küchle M, Naumann GO. Changing indications for penetrating keratoplasty: histopathology of 1,250 corneal buttons. Cornea. 1998;17(5):468-70.

17. Ghosheh FR, Cremona FA, Rapuano CJ, Cohen EJ, Ayres BD, Hammersmith KM, et al., Trends in penetrating keratoplasty in the United States 1980-2005. Int Ophthalmol. 2008;28(3):147-53.

18. Erdurmus M, Yildiz EH, Abdalla YF, Hammersmith KM, Rapuano CJ, Cohen EJ. Contact lens related quality of life in patients with keratoconus. Eye Contact Lens. 2009;35(3):123-7.

19. Hu CY, Tung HC. Managing keratoconus with reverse-geometry and dual-geometry contact lenses: a case report. Eye Contact Lens. 2008;34(1):71-5.

20. Wagner H, Barr JT, Zadnik K. Collaborative Longitudinal Evaluation of Keratoconus (CLEK) Study: methods and findings to date. Cont Lens Anterior Eye. 2007;30(4):223-32.

21. McMonnies CW. Keratoconus fittings: apical clearance or apical support? Eye Contact Lens. 2004;30(3):147-55.

22. Jankov II MR, Hafezi F, Beko M, Ignjatovic Z, Djurovic B, Markovic V, Schor P. Ultra B2 - Promoção de ligações covalentes do colágeno corneal (Corneal cross-linking) no tratamento de ceratocone: resultados preliminares. Arq Bras Oftalmol. 2008;71(6):813-8.

23. Lamy R, Netto CF, Pecego MG, Pecego JGC, Pereira BB, Moraes Júnior HV, Dantas AM. Reticulação do colágeno corneano com radiação ultravioleta e riboflavina para tratamento do ceratocone: resultados preliminares de um estudo brasileiro. Rev Bras Oftalmol. 2008;67(5):231-5.

24. Wollensak G, Spoerl E, Seiler T. Riboflavin/ultraviolet-a-induced collagen crosslinking for the treatment of keratoconus. Am J Ophthalmol. 2003;135(5):620-7.

25. Iovieno A, Oechsler RA, Yoo SH. Long-term results of collagen crosslinking with riboflavin and UVA in keratoconus. J Cataract Refract Surg. 2008;34(10):1616-7; author reply 1617.

26. Piñero DP, Alio JL, El Kady B, Coskunseven E, Morbelli H, Uceda-Montanes A, et al. Refractive and aberrometric outcomes of intracorneal ring segments for keratoconus: mechanical versus femtosecond-assisted procedures. Ophthalmology. 2009;116(9):1675-87.

27. Alió JL, Shabayek MH. Intracorneal asymmetrical rings for keratoconus: where should the thicker segment be implanted? J Refract Surg. 2006;22(3):307-9.

28. Colin J, Simonpoli-Velou S. The management of keratoconus with intrastomal corneal rings. Int Ophthalmol Clin. 2003;43(3):65-80

29. Siganos D, Ferrara P, Chatzinikolas K, Bessis N, Papastergiou G. Ferrara intrastromal corneal rings for the correction of keratoconus. J Cataract Refract Surg. 2002;28(11):1947-51.

30. Colin J, Cochener B, Savary G, Malet F. Correcting keratoconus with intracorneal rings. J Cataract Refract Surg. 2000;26(8):1117-22. Comment in: J Cataract Refract Surg. 2000;26(8):1099-100. J Cataract Refract Surg. 2001;27(3):341.

31. Kwitko S, Severo NS. Ferrara intracorneal ring segments for keratoconus. J Cataract Refract Surg. 2004;30(4):812-20.

32. Leccisotti A, Fields SV. Angle-supported phakic intraocular lenses in eyes with keratoconus and myopia. J Cataract Refract Surg. 2003;29(8):1530-6.

33. Leccisotti A. Bioptics: where do things stand? Curr Opin Ophthalmol. 2006;17(4):399-405.
34. Kymionis GD, Kontadakis GA, Kounis GA, Portaliou DM, Karavitaki AE, Magarakis M, et al. Simultaneous topography-guided PRK followed by corneal collagen cross-linking for keratoconus. J Refract Surg. 2009;25(9):S807-11.

35. Kanellopoulos AJ. Comparison of sequential vs same-day simultaneous collagen cross-linking and topography-guided PRK for treatment of keratoconus. J Refract Surg. 2009;25(9):S812-8.

36. Lin DT, Holland SR, Rocha KM, Krueger RR. Method for optimizing topography-guided ablation of highly aberrated eyes with the ALLEGRETTO WAVE Excimer Laser. J Refract Surg. 2008;24(4):S439-45.

37. Cennamo G, Intravaja A, Boccuzzi D, Marotta G, Cennamo G. Treatment of keratoconus by topography-guided customized photorefractive keratectomy: two-year follow-up study. J Refract Surg. 2008;24(2):145-9.

38. Kanellopoulos AJ, Binder PS. Collagen cross-linking (CCL) with sequential topography-guided PRK: a temporizing alternative for keratoconus to penetrating keratoplasty. Cornea. 2007;26(7):891-5.

39. Klyce SD. Computer-assisted corneal topography. High-resolution graphic presentation and analysis of keratoscopy. Invest Ophthalmol Vis Sci. 1984;25(12):1426-35.

40. Rabinowitz YS, Garbus J, McDonnell PJ. Computer-assisted corneal topography in family members of patients with keratoconus. Arch Ophthalmol. 1990;108(3):365-71.

41. Wilson SE, Lin DT, Klyce SD. Corneal topography of keratoconus. Cornea. 1991;10(1):2-8.

42. Ambrósio R Jr, Klyce SD, Wilson SE. Corneal topographic and pachymetric screening of keratorefractive patients. J Refract Surg. 2003;19(1):24-9.

43. Wolf A, Abdallat W, Kollias A, Frohlich SJ, Grueterich M, Lackerbauer CA. Mild topographic abnormalities that become more suspicious on Scheimpflug imaging. Eur J Ophthalmol. 2009;19(1):10-7.

44. Ambrósio R Jr, Alonso RS, Luz A, Coca Velarde LG. Cornealthickness spatial profile and corneal-volume distribution: tomographic indices to detect keratoconus. J Cataract Refract Surg. 2006;32(11):1851-9.

45. de Sanctis U, Loiacono C, Richiardi L, Turco D, Mutani B, Grignolo FM. Sensitivity and specificity of posterior corneal elevation measured by Pentacam in discriminating keratoconus/subclinical keratoconus. Ophthalmology. 2008;115(9):15349. Comment in: Ophthalmology. 2009;116(4):816, 816.e1; author reply 816-7.

46. Swartz T, Marten L, Wang M. Measuring the cornea: the latest developments in corneal topography. Curr Opin Ophthalmol. 2007;18(4):325-33.

47. Fontes BM, Ambrósio R Jr, Alonso RS, Jardim D, Velarde GC, Nosé W. Corneal biomechanical metrics in eyes with refraction of -19.00 to $+9.00 \mathrm{D}$ in healthy Brazilian patients. J Refract Surg.2008;24(9):941-5.

48. Fontes BM, Ambrósio R Jr, Salomão M, Velarde GC, Nosé W. Biomechanical and Tomographic Analysis of Unilateral Keratoconus. J Refract Surg. 2009:1-5. [Epub ahead of print]

49. Luce DA. Determining in vivo biomechanical properties of the cornea with an ocular response analyzer. J Cataract Refract Surg. 2005;31(1):156-62.

50. Dupps WJ Jr. Hysteresis: new mechanospeak for the ophthalmologist. J Cataract Refract Surg. 2007;33(9):1499-501. Comment on: J Cataract Refract Surg. 2007;33(9):1530-8.

51. Kotecha A. What biomechanical properties of the cornea are relevant for the clinician? Surv Ophthalmol. 2007;52 Suppl 2:S109-14. 
52. Shah S, Laiquzzaman M, Bhojwani R, Mantry S, Cunliffe I. Assessment of the biomechanical properties of the cornea with the ocular response analyzer in normal and keratoconic eyes. Invest Ophthalmol Vis Sci. 2007;48(7):3026-31.

53. Vinciguerra P, Albè E, Trazza S, Rosetta P, Vinciguerra R, Seiler T, Epstein D. Refractive, topographic, tomographic, and aberrometric analysis of keratoconic eyes undergoing corneal cross-linking. Ophthalmology. 2009;116(3):369-78. Comment in: Ophthalmology. 2009;116(10):2036-7; author reply 2037-8.

54. Schlegel Z, Lteif Y, Bains HS, Gatinel D. Total, corneal, and internal ocular optical aberrations in patients with keratoconus. J Refract Surg. 2009;25(10 Suppl):S951-7.

55. Dai GM. Wavefront reconstruction methods. J Refract Surg. 2009;25(1):9; author reply 10-1.

56. Okamoto C, Okamoto F, Samejima T, Miyata K, Oshika T. Higher-order wavefront aberration and letter-contrast sensitivity in keratoconus. Eye (Lond). 2008;22(12):1488-92.

57. Jafri B, Li X, Yang H, Rabinowitz YS. Higher order wavefront aberrations and topography in early and suspected keratoconus. J Refract Surg. 2007;23(8):774-81. Comment in: J Refract Surg. 2008;24(5):460; author reply 461-2.

58. Mihashi T, Hirohara Y, Bessho K, Maeda N, Oshika T, Fujikado T. Intensity analysis of Hartmann-Shack images in cataractous, keratoconic, and normal eyes to investigate light scattering. Jpn J Ophthalmol. 2006;50(4):323-33.

59. Marcos S. Aberrometry: basic science and clinical applications. Bull Soc Belge Ophtalmol. 2006;(302):197-213.

60. Schwiegerling J. Modal reconstruction methods with Zernike polynomials. J Refract Surg. 2005;21(5):S552-7.

61. Yoon G, Pantanelli S, MacRae S. Comparison of Zernike and Fourier wavefront reconstruction algorithms in representing corneal aberration of normal and abnormal eyes. J Refract Surg. 2008;24(6):582-90. Comment in: J Refract Surg. 2009;25(1):9; author reply 10-1. J Refract Surg. 2009;25(1):910 ; author reply $10-1$.

62. Koch DD, Wang L, Chernyak DA. Wavefront reconstruction methods. J Refract Surg. 2009;25(1):9-10; author reply 10-1.

63. Negishi K, Kumanomido T, Utsumi Y, Tsubota K. Effect of higher-order aberrations on visual function in keratoconic eyes with a rigid gas permeable contact lens. Am J Ophthalmol. 2007;144(6):924-9.

64. Lim L, Wei RH, Chan WK, Tan DT. Evaluation of higher order ocular aberrations in patients with keratoconus. J Refract Surg. 2007;23(8):825-8.

65. Kosaki R, Maeda N, Bessho K, Hori Y, Nishida K, Suzaki A, et al. Magnitude and orientation of Zernike terms in patients with keratoconus. Invest Ophthalmol Vis Sci. 2007;48(7):3062-8.

66. Maeda N, Fujikado T, Kuroda T, Mihashi T, Hirohara Y, Nishida $\mathrm{K}$, et al. Wavefront aberrations measured with HartmannShack sensor in patients with keratoconus. Ophthalmology. 2002;109(11):1996-2003.

67. McMahon TT, Szczotka-Flynn L, Barr JT, Anderson RJ, Slaughter ME, Lass JH, Iyengar SK; CLEK Study Group. A new method for grading the severity of keratoconus: the Keratoconus Severity Score (KSS). Cornea. 2006;25(7):794-800.

68. Shahh S, Naroo S, Hosking S, Gherghel D, Mantry S, Bannerjee $\mathrm{S}$, et al. Nidek OPD-scan analysis of normal, keratoconic, and penetrating keratoplasty eyes. J Refract Surg. 2003;19(2 Suppl):S255-9.

69. Chalitaa MR, Krueger RR. Wavefront aberrations associated with the Ferrara intrastromal corneal ring in a keratoconic eye. J Refract Surg. 2004;20(6):823-30.
70. Marsack JD, Parker KE, Pesudovs K, Donnelly WJ 3rd, Applegate RA. Uncorrected wavefront error and visual performance during RGP wear in keratoconus. Optom Vis Sci. 2007;84(6):463-70.

71. Sabesan R, Yoon G. Visual performance after correcting higher order aberrations in keratoconic eyes. J Vis. 2009;9(5):6.1-10.

72. Charman WN. Wavefront aberration of the eye: a review. Optom Vis Sci. 1991;68(8):574-83.

73. Holladay JT. Visual acuity measurements. J Cataract Refract Surg. 2004;30(2):287-90.

74. Holladay JT. Proper method for calculating average visual acuity. J Refract Surg. 1997;13(4):388-91.

75. Netto MV, Dupps W Jr, Wilson SE. Wavefront-guided ablation: evidence for efficacy compared to traditional ablation. Am J Ophthalmol. 2006;141(2):360-8.

76. Partal AE, Manche EE. CustomVue laser in situ keratomileusis for myopia and myopic astigmatism using the Visx S4 excimer laser: Efficacy, predictability, and safety. J Cataract Refract Surg. 2006;32(3):475-9.

77. Stonecipher KG, Kezirian GM. Wavefront-optimized versus wavefront-guided LASIK for myopic astigmatism with the ALLEGRETTO WAVE: three-month results of a prospective FDA trial. J Refract Surg. 2008;24(4):S424-30.

78. Seiler T, Dastjerdi MH. Customized corneal ablation. Curr Opin Ophthalmol. 2002;13(4):256-60.

79. Carones F. Diagnostic use of ocular wavefront sensing. Ophthalmol Clin North Am. 2004;17(2):129-33, v.

80. Charman WN. Wavefront technology: past, present and future. Cont Lens Anterior Eye. 2005;28(2):75-92.

81. Wang L, Wang N, Koch DD. Evaluation of refractive error measurements of the Wavescan Wavefront system and the Tracey Wavefront aberrometer. J Cataract Refract Surg. 2003;29(5):970-9. Comment in: J Cataract Refract Surg. 2004;30(5):942.

82. Marsack JD, Parker KE, Applegate RA. Performance of wavefront-guided soft lenses in three keratoconus subjects. Optom Vis Sci. 2008;85(12):E1172-8.

83. Katsoulos C, Karageorgiadis L, Vasileiou N, Mousafeiropoulos $\mathrm{T}$, Asimellis G. Customized hydrogel contact lenses for keratoconus incorporating correction for vertical coma aberration. Ophthalmic Physiol Opt. 2009;29(3):321-9.

84. Pepose JS, Qazi MA, Edwards KH, Sanderson JP, Sarver EJ. Comparison of contrast sensitivity, depth of field and ocular wavefront aberrations in eyes with an IOL with zero versus positive spherical aberration. Graefes Arch Clin Exp Ophthalmol. 2009;247(7):965-73.

85. Packer M, Fine IH, Hoffman RS. Aspheric intraocular lens selection based on corneal wavefront. J Refract Surg. 2009;25(1):12-20.

86. Koch DD, Wang L. Custom optimization of intraocular lens asphericity. Trans Am Ophthalmol Soc. 2007;105:36-41; discussion 41-2.
Endereço para correspondência:
Dr. Renato Ambrósio Jr.
Rua Conde de Bonfim, $\mathrm{n}^{\circ} 211$ apto.712 - Tijuca
CEP 20520-050 - Rio de Janeiro (RJ), Brasil
E-mail: renatoambrosiojr@terra.com.br 